\title{
PARTICIPACIÓN E INTEGRACIÓN DE LA MUJER MARROQUÍ EN MADRID
}

\author{
PARTICIPATION AND INTEGRATION OF MOROCCAN WOMEN IN \\ MADRID
}

\author{
Mohamed Boundi Boundi \\ Universidad Complutense, Madrid. España/Spain \\ boundimed@gmail.com
}

Recibido/Received: 23/01/2012

Modificado/Modified: 31/03/2012

Aceptado/Accepted: 27/06/2012

\section{RESUMEN}

La mujer marroquí que vive y trabaja en la Comunidad Autónoma de Madrid es portadora de una serie de valores culturales que define su identidad como árabe y musulmana. En su sociedad de acogida, intenta adaptarse a la realidad de su nuevo entorno como madre y a veces como trabajadora y ciudadana. Es joven, independiente y dispuesta a desarrollar sus habilidades, tejar amistades con los autóctonos y adquirir nuevos conocimientos y habilidades. Su meta es convertirse en ciudadana de pleno derecho en España.

\section{PALABRAS CLAVE}

Mercado, perfil, independiente, autóctonos, acogida.

\section{ÍNDICE}

1. Introducción. 2. Radioscopia de la población marroquí en la CAM en 2010. 3. Estudio de la situación de la mujer marroquí en la CAM. 3.1. Recursos de investigación y metodología. 3.2. Determinación de la muestra. 4. Conclusiones. Bibliografía.

\begin{abstract}
The Moroccan woman who lives and works in the Autonomous Community of Madrid is a carrier of a series of cultural values that define her identity as an Arab and Muslim. In her host society, she is trying to the reality of her new environment as mother sometimes as a worker and citizen. She's young, independent and willing to develop skills, friendships with native tile and acquire new knowledge and skills. Their aim is to become full citizens in Spain.
\end{abstract}

\section{KEYWORDS}

Market, profile, independent, autochthonous, host.

\section{CONTENTS}

1. Introduction. 2. Radioscopy of the Moroccan population in the CAM in 2010. 3. Study of the status of the Moroccan women in la CAM. 3.1. Research resources and methodology. 3.2. Determination of the simple. 4. Conclusions. References. 


\section{INTRODUCCIÓN}

El objetivo de esta investigación consiste en acercarnos a la condición humana de la mujer marroquí en la Comunidad Autónoma de Madrid (CAM). Constituye, también, una oportunidad de conocer su situación como ama de casa, trabajadora fuera del hogar, esposa o canal de transmisión de valores culturales. En definitiva, forma parte del conjunto de los estudios, trabajos de campo y publicaciones que intentan sensibilizar a la opinión pública española sobre la necesidad de promover el mestizaje cultural.

Actualmente, España está en plena mutación social por la masiva incorporación en la sociedad, desde los años 1990, de diversas culturas, etnias y razas. Desde la promulgación en 1985 de la primera Ley de Extranjería (BOE, 1985), las Cortes modificaron en 2000, 2003 y 2009 este texto legislativo que regula las condiciones de estancia y de trabajo de los extranjeros en España. En abril de 1991, el Congreso de los Diputados aprobó una Proposición no de ley invitando al Gobierno a revisar la política migratoria. La revisión el 11 de diciembre de 2009 de la Ley Orgánica sobre derechos y libertades de los extranjeros en España y su integración social (BOE, 2009), fue el último intento de desarrollar el contenido de este texto.

En el plano académico, se desarrollan conceptos y planteamientos acerca del modelo de sociedad que se necesita en España. Aunque existe una evidencia empírica según la cual las sociedades multiculturales son un hecho real en un mundo globalizado (Abella, 2003), en España se denota, a nivel teórico, una ignorancia del "otro". Esta actitud se justifica por el hecho de que la sociedad española no estaba preparada, al principio, para acoger, en un corto periodo, una numerosa inmigración de distintas culturas. También ha nacido un debate acerca del valor de la economía de empresa, cuyo propósito es la creación de "actividades industriales, mercantiles o de prestación de servicios con fines lucrativos" en las que intervienen, también, las inmigrantes (Real Academia Española, 2009). Este concepto ha evolucionado desde el momento en que las amas de casa españolas se incorporan masivamente al mercado laboral para realizar actividades o profesiones lucrativas.

La dificultad en el planteamiento de la problemática de la integración de la mujer marroquí en la sociedad española surge cuando abordamos su condición de trabajadora, madre/esposa y sostén económico. Su desclasamiento en la sociedad de acogida (Martínez Veiga, 2004) y el chantaje al que está sometida, a veces, en la sociedad de origen laminan su autoestima.

Son algunos factores que condicionan la convivencia en la sociedad de acogida, la esfera privada de la mujer marroquí y la cultura grupal. No obstante, será útil delimitar el campo de nuestra investigación para poder conocer los motivos que incitan a la mujer marroquí a emigrar. Más allá de este objetivo general, hay otro específico que va a permitirnos conocer el perfil de la mujer marroquí en la CAM, sus inquietudes y sus expectativas. Una encuesta socio-laboral nos aportará la respuesta a nuestro afán de acercarnos al mundo de las inmigrantes marroquíes en la Comunidad Autónoma de Madrid.

\section{RADIOSCOPIA DE LA POBLACIÓN MARROQUÍ EN LA CAM EN 2010}

Para conseguir una radioscopia próxima a la realidad de la población marroquí en la CAM, es imprescindible recabar datos del Instituto Nacional de Estadística, de los afiliados a 
la Seguridad Social en 2011 y del informe demográfico de la población extranjera del Observatorio en la Comunidad de Madrid (CAM, 2011).

Hasta septiembre de 2011, vivían en España 330.366 mujeres y 496.504 hombres marroquíes (Observatorio permanente de la inmigración, 2011). Formado por 826.870 miembros $(15,91 \%$ del total de los extranjeros), el colectivo marroquí ha crecido un $4,6 \%$ en comparación con diciembre de 2010 (MEYSS, 2012).

A nivel de la CAM, consideramos que el Informe Demográfico de la Población Extranjera como el documento más completo y detallado para poder establecer una radioscopia de la población marroquí en esta región. Tiene el mérito de recapitular datos de los 179 municipios de la Comunidad de Madrid que son tratados por el INE.

Hasta junio de 2011, había en la CAM un total de 89.867 marroquíes, lo que representa el $8,45 \%$ del total de los 1.062 .817 extranjeros.

La inmigración marroquí es, mayoritariamente, masculina puesto que está compuesta por $58 \%$ de hombres y $42 \%$ de mujeres, un índice ligeramente inferior al que hemos calculado a nivel nacional (60\% por $40 \%$ respectivamente).

Es también una población desigualmente repartida en el territorio de la CAM. Aunque en su mayoría se concentra en Madrid capital; en la Corona Sur y la Corona Oeste está representada en 161 de los 179 municipios (89,38\%). Pese a su importancia numérica, sorprende que sólo cuatro municipios tengan más de 5.000 marroquíes empadronados.

En un único municipio, Madrid, nos encontramos con una concentración de 26.476 marroquíes. Sin embargo, este colectivo se posiciona lejos de las demás nacionalidades mayoritarias como la ecuatoriana (87.108), la rumana (63.693), la peruana (40.031), la boliviana (38.830) o la colombiana (35.999).

El análisis de los datos provisionales del INE demuestra particularmente una fuerte dispersión de los inmigrantes marroquíes y que en ninguna área geográfica de la CAM figuran a la cabeza de la lista de las nacionalidades mayoritarias. Su grueso se concentra en Madrid capital $(28,86 \%)$ y la Corona sur $(27,9 \%)$. Una cuarta parte vive en la zona no metropolitana (24,98\%), sobre todo en la zona Sur Oeste.

La presencia marroquí es casi nula en algunos municipios, como en el caso de treinta municipios que cuentan con menos de 20 habitantes de esta nacionalidad. En total, el $90 \%$ de los municipios cuenta con menos de 1.000 habitantes de origen marroquí. La Corona Metropolitana Sur es la única zona donde Marruecos destaca como la nacionalidad Mayoritaria $(17,77 \%)$. En la Corona Metropolitana Oeste, la presencia marroquí es mayoritaria en dos municipios, Galapagar (23,29\%) y Collado Villalba (18,67\%).

$\mathrm{Al}$ analizar los datos recopilados sobre las categorías de edad de la población marroquí, constatamos que se trata de un colectivo joven. Sobre el total de los 91.754 marroquíes en la CAM en 2010, el 77\% tiene menos de 40 años mientras los empadronados de más de 50 años suman un $8 \%$. Estos datos confirman el alto índice de marroquíes en edad de ejercer una actividad lucrativa, formarse o contribuir en la mejora del bienestar de su familia. Los que tienen menos de 20 años representan la cuarta parte del total, la mayoría tiene menos de 10 años (17,76\%). Sólo el 2\% tiene más de 60 años.

En comparación con los demás colectivos de inmigrantes en la CAM, la población marroquí es la más masculinizada (58\%) puesto que el número de hombres es superior al de las mujeres en valores absolutos, 15.138 miembros.

Aparte del censo demográfico y del padrón municipal, había a nivel nacional 208.712 marroquíes dados de alta en la Seguridad Social (Seguridad Social, 2012) hasta diciembre de 2011, dentro de ellos 22.300 ejercen en Madrid. 
En 2011, el 10,93\% del colectivo de los inmigrantes marroquíes en España vive, trabaja o estudia en condiciones legales en la CAM. En este contexto, señalamos que la proporción de las mujeres ha crecido en casi tres puntos en la CAM en comparación con el año 2009. EN el ámbito nacional, el $24,56 \%$ del colectivo marroquí está afiliado a la $\mathrm{SS}$, hasta finales de diciembre de 2011.

\section{ESTUDIO DE LA SITUACIÓN DE LA MUJER MARROQUÍ EN LA CAM}

\subsection{Recursos de investigación y metodología}

El objetivo específico de este ensayo consiste en conocer a las mujeres marroquíes que viven, trabajan o se dedican exclusivamente a la dirección del hogar familiar en la ciudad de Madrid. El principal recurso para alcanzar este objetivo es una encuesta sociológica. Debido a las características de nuestro trabajo que se dedica a la comunidad de los inmigrantes en Madrid y los medios materiales de que disponemos, aspiramos únicamente a esbozar un perfil aproximativo de la mujer marroquí, sus principales preocupaciones, percepciones de la sociedad de acogida, relaciones cotidianas tanto en la calle como en el trabajo o con su nuevo entorno social. Con el ánimo de acercarnos a la mujer marroquí, como ciudadana, trabajadora o madre, el cuestionario ha sido preparado en colaboración con los mediadores sociales de la Asociación Solidaria para la Integración Sociolaboral del Inmigrante (ASISI) debido a su permanente contacto con los inmigrantes de distintas nacionalidades.

Nos hemos inspirado en trabajos de institutos de investigación (CIS, 2007), sociólogos (Tezanos y Díaz Moreno, 2008) y universitarios (Soriano Miras y Santos Bailón, 2002) que están al alcance del gran público. Nuestra preocupación consiste en presentar una descripción más próxima a la condición de la mujer marroquí en la sociedad de acogida.

Por tal razón, el cuestionario ha sido estructurado en bloques de manera que podemos reunir el máximo de datos en relación con su estatus en el nuevo entorno socio-laboral sin la intención de exigirle desvelar aspectos de su vida íntima por no ser la finalidad de este estudio. De modo que el cuestionario ha sido concebido en función de problemas planteados cada día en los contactos entre los mediadores sociales y los inmigrantes. Las preguntas son formuladas en un sencillo lenguaje que permite a la entrevistada dar una respuesta correcta. Además, la comunicación es bilingüe, en árabe y español (a veces trilingüe si añadimos el idioma berebere, el amazig) por el hecho de que la encuesta está dirigida sólo a marroquíes. El árabe es, en este contexto, un componente primordial en la comunicación, y se convierte para las entrevistadas en un elemento de seguridad, de proximidad y de facilidad para lanzar una respuesta espontánea. El papel que desempeñan los encuestadores es imprescindible, en este caso, por tener la ventaja de dominar tres idiomas (árabe, berebere y español) y crear un ambiente de confianza para la comprensión de las preguntas.

\subsection{Determinación de la muestra}

Las entrevistas han sido realizadas exclusivamente en la sede de ASISI durante dos meses (junio y julio de 2010) con las usuarias que acudían a esta asociación para la busca de empleo, consultas diversas o con las beneficiarias de los servicios prestados, enseñanza del idioma o clases de informática. En el diseño de la muestra se ha fijado como meta entrevistar a todas las marroquíes con edades superiores a los 18 años. De las 46 preguntas de la encuesta, agrupadas en 14 variables, hemos conseguido formar siete bloques o categorías.

El cuestionario consta de 46 preguntas agrupadas en ocho bloques o categorías: 
1. Perfil: hemos incorporado en este bloque seis variables para conocer el estado civil de la entrevistada, la región de origen en Marruecos, el medio de transporte usado para llegar a España, los vínculos familiares en Marruecos y la situación familiar en España.

2. Situación administrativa y laboral: este bloque comporta datos sobre la duración de residencia o estancia en España, y para saber si la entrevistada tiene la nacionalidad española, el premiso de trabajo y de residencia, tiene sólo la tarjeta de residencia y no el permiso de trabajo, si está indocumentada, en trámites de regularización u otra situación (como casada con un comunitario).

3. Aspectos profesionales y laborales: se pretende conocer en este bloque si la entrevistada trabaja en la actualidad y cuanto tiempo ha trabajado desde su llegada a España.

4. Ocupaciones: bloque que enumera los sectores ocupacionales en los que pueda ejercer la entrevistada y si, en caso de trabajar cara al público, fue objetivo de amenazas, insultos, acoso sexual o discriminación por su vestimenta, raza o cultura.

5. Ambiente familiar: procuramos saber en este bloque, cómo la entrevistada trabajadora compagina su vida laboral con sus responsabilidades de madre y esposa, si su marido colabora en las tareas domésticas, si está dispuesta a dejar su trabajo por cuidar a sus niños o si beneficia de una determinada ayuda o prestación social.

6. Aspectos educativos, culturales y de conocimiento de la sociedad española: Los datos recabados en este bloque anticipan al conocimiento de la capacidad de la entrevistada de integrarse en la sociedad española. Tres variables son escogidas para medirlo: el manejo del idioma español, el nivel cultural y los conocimientos en la cultura general de la entrevistada.

7. Aspectos relacionados con la participación ciudadana: es la categoría más importante del trabajo de terreno con 19 preguntas que abrazan elementos que puedan influir en el comportamiento de la mujer marroquí dentro de la sociedad de acogida. La entrevistada tiene la oportunidad de sincerarse para opinar sobre la actitud de los españoles, la imagen de los marroquíes, la religiosidad, el consumo de los canales de televisión españoles y marroquíes, la adicción a algún tipo de drogas o la práctica de deporte.

8. Grado de satisfacción de la convivencia en España: este bloque comprende una sola pregunta cerrada que resume en una palabra las intenciones de la entrevistada de poner fin a su proyecto migratorio o, al contrario, quedarse en España.

\section{CONCLUSIONES}

El análisis de las 204 entrevistas realizadas ha permitido sacar unas conclusiones que van a facilitar la determinación del perfil de la mujer marroquí en la CAM. Como premisa, se trata de una mujer que vive en las mismas condiciones que las demás inmigrantes por ser a la vez madre, trabajadora y casada o soltera.

1. Perfil: La mujer marroquí, instalada en la CAM, es joven, soltera y proviene del Norte de Marruecos. Es su perfil general.

2. Edad: Aunque es muy reducida, nuestra encuesta desvela la presencia de una alta proporción de mujeres marroquíes jóvenes en la pirámide demográfica de España. Casi la mitad de las mujeres entrevistadas (45\%) se encuentra en la categoría de edad de 30-39 años, un dato que demuestra la juventud de la mano de obra femenina marroquí y su preparación física para desempeñar cualquier tipo de tareas. La misma observación es válida cuando analizamos la categoría de edad de menos de 30 años que representa más del tercio de las 
mujeres entrevistadas (35\%). En esta categoría se congregan las estudiantes, las hijas de inmigrantes, las casadas recién reagrupadas o las hijas de inmigrantes que forman la segunda generación. El colectivo femenino marroquí en la CAM, objeto de nuestro estudio, se distingue por su juventud, cuando la población autóctona y la de Marruecos sufren un progresivo proceso de envejecimiento. Nos encontramos en presencia de un transvase demográfico a favor de España, una operación habitual en los movimientos migratorios.

3. Estado civil: La mujer marroquí prefiere vivir independiente $(58 \%)$ del entorno familiar y no manifiesta ninguna voluntad de unir su destino al de un hombre en su proyecto migratorio. Además, la proporción de las mujeres que ha dejado un cónyuge/pareja en Marruecos es menor (42\%). No obstante, las "solteras" son las más numerosas dentro de esta categoría $(29 \%)$, las divorciadas o separadas $(22 \%)$ y las viudas $(7 \%)$ completan este abanico.

4. Zona de procedencia: el $46 \%$ de ellas proviene del Norte de Marruecos. Este dato permite también verificar la hipótesis de la influencia cultural española en esta región. Tradicionalmente, los intercambios humanos y culturales entre las dos orillas del Estrecho de Gibraltar son fuertes y diversos. Los habitantes del Norte de Marruecos mantienen contactos diarios con los españoles a través del comercio directo, de la inmigración circular, la facilidad de acceso a los medios audiovisuales españoles y el dominio del idioma por una amplia franja de la población de la zona.

5. Adicciones: podemos admitir, a tenor de los resultados conseguidos, que las mujeres marroquíes en la CAM no constituyen una carga ni para los servicios sociales ni para los servicios de salud en cuanto al tratamiento de enfermedades derivadas del consumo de tabaco, del alcohol o de drogas. Son personas sanas que fuman muy poco, no beben ni son ludópatas. Aunque viven en un país occidental, siguen estando muy influidas por su cultura arabe-musulmana. El Islam prohíbe tajantemente el consumo de alcohol y los juegos de azar y apuestas. En su país de acogida, se desarrollan campañas de sensibilización contra el tabaco y los perjuicios que pueda causar su consumo para la salud. En nuestra encuesta, sólo 12 mujeres reconocen fumar y una sola confiesa beber alcohol. De las 204 entrevistadas ninguna declara frecuentar los lugares de juegos, casinos o echar apuestas de loterías. Según la Encuesta Europea de Salud en España del Instituto Nacional de Estadística (INE), publicada a finales de 2009, un 22,2\% de las mujeres en España suele fumar a diario y 3,9\% de forma puntual (INE, 2009). En otra encuesta, elaborada por el mismo organismo en 2007, el 66,4\% de la población femenina de España había consumido alcohol a lo largo de ese año (INE, 2007). En su barómetro de diciembre de 2009, el CIS indica que el $26 \%$ de las personas entrevistas de distintas nacionalidades juega a la lotería en Navidad y alguna vez más a lo largo del año, y el 7,8\% lo hace todas o casi todas las semanas (CIS, 2009).

6. Deporte: apenas practican deportes. La baja proporción (14\%) de las marroquíes que suelen acudir al gimnasio o hacer habitualmente ejercicios físicos muchas veces a la semana, se justifica por la falta de hábitos, los largos horarios de trabajo y por el bajo poder adquisitivo. La mayoría de ellas son empleadas de hogar y pocas de ellas disfrutan habitualmente de un descanso semanal. Más de una cuarta parte (29\%) de las mujeres afirma practicar "poco" deporte, lo que es a su entender, caminar más de una hora cada día o hacer carrera suave (footing) cada fin semana. Sin embargo, la mayoría (57\%) admite su condición de sedentarias por no haber hecho nunca ejercicios físicos.

7. Vínculos familiares en Marruecos: aunque gozan de un arraigo social, por llevar muchos años en España, la mayoría de ellas mantiene fuertes lazos con su familia en Marruecos por haber dejado a padres o confiado la educación de sus hijos a familiares. 
Además, el peso de la cultura y los lazos sanguíneos actúan como códigos morales en las relaciones de familia. Las madres, que han dejado hijos en su país (13\%) o esposos (7\%), se comprometen a mantener unos fluidos y sólidos lazos con ellos. Por la cercanía geográfica y el bajo coste de los transportes, estos lazos se renuevan continuamente mediante las periódicas visitas a lo largo del año, amén del envío de remesas. Además el $80 \%$ afirma tener padres en el país.

8. Vínculos familiares en España: debido a las dificultades de adaptación en su nuevo ámbito, la mayoría (63\%) no tiene todavía hijos en España. Por llegar a España mediante el proceso de reagrupación familiar y carecer de permiso de trabajo, se afrontan desde el principio a la dificultad de acceder a un empleo estable. El 19\% afirma vivir bajo el mismo techo con hijos y pareja, un dato que demuestra la baja proporción de familias tradicionales constituidas de padre, madre e hijos. El porcentaje de familias monoparentales es aun bajo $(18 \%)$. Se trata en este caso de mujeres viudas o separadas.

9. Llegada a España: El 83\% de las mujeres marroquíes vino a instalarse en la CAM por vías regulares. La reagrupación familiar se refleja como el principal canal que permitió a la mitad de ellas acceder a una situación legal como inmigrantes. Sólo el 11\% reconoce haber llegado a España de manera irregular sin especificar el modo usado. Otras pasaron por los canales institucionalizados por los gobiernos de Madrid y Rabat a favor de la mano de obra marroquí como los cupos anuales o contingentes temporales (11\%). Sólo 1\% de las mujeres afirma llegar a Madrid mediante una autorización de estancia por estudios.

10. Situación administrativa y laboral: las mujeres marroquíes en la CAM están en situación regular.

La casi totalidad de las mujeres marroquíes (91\%) lleva más de dos años de estancia y tiene los papeles en regla. Se reparten en tres categorías: las que tienen el permiso de trabajo y la tarjeta de residencia (64\%), las que poseen sólo una tarjeta de residencia $(16 \%)$, y, las que tienen la nacionalidad (5\%), están en tramites de regularización $(5 \%)$ o en otras situaciones (1\%: tarjeta comunitaria). Las "sin papeles" representan únicamente el 9\% del colectivo femenino marroquí. Además, el número de marroquíes que acceden a la nacionalidad española crece cada año.

Más de la mitad de las mujeres marroquíes estaba sin actividad (59\%) en el periodo en que hicimos las encuestas. Es una proporción muy alta en comparación con la tendencia general del mercado laboral en España, donde la tasa media de paro alcanzó el 20,3\% en enero de 2011 y 22,85\% en enero de 2012, según el Instituto Nacional de Estadística (INE). Dentro de la proporción de las mujeres en actividad, el $42 \%$ lleva más de tres años trabajando mientras casi un tercio (31\%) cuenta con menos de dos años de trabajo efectivo desde su llegada a España. El 12\% declara no haber tenido todavía la oportunidad de ejercer una actividad renumerada en España, un dato que nos parece preocupante puesto que una buena proporción del colectivo femenino marroquí no tiene la oportunidad de colaborar en la mejora de su bienestar y el de su familia.

Las que ejercen una actividad remunerada, trabajan en el servicio doméstico $(55 \%)$ o cuidan a niños (14\%) y mayores (13\%), lo que representa una proporción de $83 \%$ de las mujeres entrevistadas. Este dato corrobora su condición de empleadas invisibles por no trabajar cara al público. El 14\% se reparte entre distintas actividades del tercer sector como la hostelería y las actividades de carácter administrativo. En el ejercicio de su trabajo, admiten que gozan de un correcto trato por parte de sus empleadores y del público. Por razones laborales, el 20,5\% afirma ser objeto de insultos (13 casos), algún tipo de discriminación por su raza, su vestimenta o su nacionalidad (19 casos), o acoso sexual (10 casos). 
11. Ambiente familiar: Para preservar la estabilidad familiar, la mayoría se declara confiada en el apoyo del marido para concretizar su deseo de incorporase al mercado laboral (56\%) aunque éste no manifieste la predisposición de colaborar en las tareas domésticas (39\%). En algunos casos, la mujer marroquí madre que desempeña una actividad laboral, destaca la alternativa de delegar la tarea de cuidar a sus niños a familiares (en España o en Marruecos) con una proporción global de 37\%. Este dato demuestra el fortalecimiento del sentimiento solidario dentro de la familia marroquí. El 30\% confía el niño a la guardería y el $15 \%$ no sabe qué opción tomar cuando se le presente esta situación por no tener todavía niños. Un porcentaje de $18 \%$ de este colectivo prefiere renunciar a su derecho al trabajo para poder asumir personalmente el cuidado de sus hijos en su propia casa.

11. Aspectos educativos, culturales y de conocimiento de la sociedad española: a pesar de su cultura afro-árabe, es sorprendente destacar la facilidad con la que la mujer marroquí consigue aprender el español. De modo que el 44\% afirma hablar "bien" el español y manejar perfectamente este idioma. Se trata de las mujeres que acabaron la secundaria $(22 \%)$, tienen un bachillerato (14\%) o una carrera universitaria (12\%). El resto de las entrevistadas maneja de manera "regular" (40\%) o "mal" (16\%) este idioma. En contra, la mujer marroquí padece un déficit a nivel de cultura general por manifestar su incapacidad de conocer quiénes son el alcalde de Madrid, el jefe del Estado español, el número de idiomas que se hablan en España o el autor de Don Quijote de la Mancha.

12. Aspectos relacionados con la participación ciudadana: La mujer marroquí es una persona sociable aunque no es una gran aficionada a las reuniones de grupos ni a la organización de actos sociales. Casi la mitad (44\%) participa o tuvo la oportunidad de participar a las actividades asociativas, y más de dos tercios disfrutan de amistades con los nativos $(67 \%)$ lo que les permite formar una percepción global de los comportamientos de los españoles hacia la comunidad marroquí.

13. Actitudes: para el $53 \%$ de las mujeres entrevistadas, los españoles son personas "muy solidarias" y los marroquíes gozan de una "buena imagen" dentro de la sociedad española. Sólo el 1\% piensa que algunos españoles adoptan actitudes "intolerantes y racistas", un dato que se deduce de la experiencia personal de cada entrevistada. En relación con la imagen que tienen los españoles de los marroquíes, el 58\% de ellas piensa que es positiva frente al 39\% que piensa lo contrario. La distancia que les separa de su patria no les impide, sin embargo, preservar sus señas de identidad como musulmana "muy practicante" $(69 \%)$ que celebra con fervor las fiestas religiosas de su país $(93 \%)$. Para manifestar su grado de integración en su nuevo entorno social, comparte con los españoles la celebración de algunas de sus fiestas (28\%) y anima a sus hijos a ampliar la red de sus amistades a los niños nativos $(80 \%)$.

En el contexto del matrimonio mixto, las mujeres adoptan dos actitudes antagonistas: el $36 \%$ lo toleran frente al 39\% que lo rechazan. Queda un 25\% que prefiere no pronunciarse. La mujer marroquí le importa mucho ver las cadenas de televisión tanto españolas como marroquíes y árabes (55\%). Es el principal medio de entretenimiento por la amplia oferta de canales y el fácil uso de los medios de recepción (antenas parabólicas) de cadenas de habla árabe y francés.

14. Grado de satisfacción de la convivencia en España: la mujer marroquí se declara satisfecha de su estancia en la CAM. Más de un tercio de las 204 mujeres entrevistadas (36\%) afirman estar satisfechas de su estancia y expresan su deseo de quedarse para siempre en España; mientras que el $27 \%$ manifiesta su intención de regresar a su país de origen. No obstante, la proporción más alta, el $37 \%$, afirma no haber tomado todavía una decisión al 
respecto. De modo que sólo una cuarta parte ha decidido regresar a su país una vez cumplido su proyecto migratorio. La coyuntura actual, que tiene una fuerte incidencia en el colectivo marroquí, el más castigado por la crisis económica, influye de manera decisiva en el comportamiento de las mujeres marroquíes que están en el paro y sin claras perspectivas de futuro. Aún así, muchas de ellas que llevan bastantes años en España, reúnen los requisitos exigidos para obtener la nacionalidad española.

El análisis de las variables ayuda a establecer una idea global sobre el comportamiento de este colectivo. En general, reúne todas las condiciones para fortalecer el arraigo de sus hijos, mejorar su cultura general e incorporarse al mercado laboral. Es también, un colectivo que intenta sacar provecho de su disponibilidad de comunicar con los nativos en su propio idioma, adaptarse a todo tipo de actividades en el mercado laboral y animar a sus hijos a ampliar la red de amistades en su entorno.

En el afán de acercarse a los valores culturales de los autóctonos, las mujeres marroquíes suelen ver los programas televisivos tanto españolas como árabes y comparten con los nativos la celebración de sus fiestas. En ningún caso expresan actitudes de rechazo hacia los usos y costumbres del país de acogida. Tampoco se encierran en sí mismas para rechazar el mestizaje cultural. Al contrario, desempeñan actividades en lugares íntimos y de privacidad que exigen cariño, afección y cordialidad como el empleo doméstico o el cuidado de los niños y mayores.

El resultado es muy llamativo, respecto a sus amistades, por el hecho de que, casi el $70 \%$ gozan de amistades con los nativos y $36 \%$ admite el matrimonio mixto entre sus hijos y los nativos.

\section{BIBLIOGRAFÍA}

ABELLA, C. M. (2003), "Globalización y multiculturalismo: ¿son posibles las democracias multiculturales en la era del globalismo?" Scripta Nova, Universidad de Barcelona, 15 de febrero, vol. VII, núm. 135, en http://www.ub.es/geocrit/sn/sn-135.htm, visitado 29/1/2012.

BOE (2009), "Ley Orgánica 2/2009, de 11 de diciembre, de reforma de la Ley Orgánica 4/2000, de 11 de enero, sobre derechos y libertades de los extranjeros en España y su integración social", 12 de diciembre, $\mathrm{n}^{\circ} 299$.

BOE (1985) "Ley Orgánica 7/1985, de 1 de julio, sobre derechos y libertades de los extranjeros en España", 03 de Julio 1985, $\mathrm{n}^{\circ} 0158$.

CAM (2011), "Informe demográfico de la población extranjera en la Comunidad de Madrid, junio 2011", en http://www.madrid.org, visitado 6/1/2011.

CIS (2009), "Barómetro de diciembre de 2009”, n 2.824, en http://datos.cis.es/pdf/Es2824mar_A.pdf, visitado $26 / 1 / 2012$.

CIS (2007), "Actitudes ante la discriminación por origen racial o étnico", estudio $n^{\circ} 2731$ en www.cis.es/cis/opencms/-Archivos/Marginales/2720_2739/2731/Ft2731.pdf, visitado 26/1/2012.

EL MUNDO (2010), "España nacionaliza casi 100 inmigrantes al día desde hace una década”, 23/2, en http://www.elmundo.es/elmundo/2010/02/23/espana/1266925123.html, visitado 16/10/2010.

HAUT COMMISSARIAT AU PLAN (2012), "Taux de Chômage selon le sexe", en http://www.hcp.ma/Taux-de-Chomage-selon-le-sexe_a256.html, visitado 13/1/2012.

INE (2010), "Nota de Prensa: Avance del Padrón municipal a 1 de enero de 2010. Datos provisionales, en http://www.ine.es/prensa/np595.pdf, visitado 19/1/2012.

INE (2009), "Datos de la encuesta Europea de Salud en 2009”, en http://www.ine.es/prodyser/ pubweb/espcif/espcif10.pdf, visitado 31/1/2012.

INE (2007), "Consumo de drogas por frecuencia, tipo de sustancia psicoactiva, periodo y sexo", en 
http://www.ine.es/jaxi/tabla.do, visitado 31/1/2012.

MARTÍNEZ VEIGA, U. (2004), Trabajadores invisibles. Precariedad, Rotación y Pobreza de la inmigración en España. Madrid: Catarata.

MINISTERIO DE EMPLEO Y SEGURIDAD SOCIAL (2012), "Resumen de datos", en http://www.meyss.es/es/estadisticas/resumenweb/RUD.pdf, visitado 29/1/2012.

REAL ACADEMIA ESPAÑOLA (2009), Diccionario de la Lengua Española, en http://www.rae.es/, visitado 5/10/2009.

SEGURIDAD SOCIAL (2010), “Afiliación”, en http://www.segsocial.es/Internet_1/Estadistica/Est /aaa/Afiliaciones en alta laboral/AplicacionesPub, visitado 14/7/2010.

SORIANO MIRAS, R. M. y SANTOS BAILÓN, C. (2002), "El perfil social de la mujer inmigrante marroquí en España y su incidencia en la relación intercultural", Papeles de Geografía, $\mathrm{n}^{\circ}$ 36, pp. 171184.

TEZANOS, J. F. y DÍAZ MORENO, V. (2008), Condiciones laborales de los trabajadores inmigrantes en España. Madrid: Sistema.

\section{Breve currículo:}

\section{Mohamed Boundi Boundi}

Es doctor en Sociología y Ciencias de la Comunicación y licenciado en Periodismo. Autor de Sin Ellas no se mueve el mundo, La mujer marroquí en la CAM: participación y convivencia y EspañaMarruecos: Heridas sin cicatrizar, es también presidente del Círculo de Investigación para la Inmigración, el Desarrollo y la Cooperación (CIIDYC). 